\title{
Bundle Folding Type III Bricard Linkages
}

\author{
Shengnan Lyu ${ }^{\mathrm{a}, \mathrm{c}}$, Dimiter Zlatanov ${ }^{\mathrm{b}, *}$, Matteo Zoppi ${ }^{\mathrm{b}}$, Xilun Ding ${ }^{\mathrm{a}}$, Gregory S. Chirikjian ${ }^{\mathrm{c}}$, Simon \\ D. Guest ${ }^{\mathrm{d}}$ \\ ${ }^{a}$ Beihang University, School of Mechanical Engineering \& Automation \\ ${ }^{b}$ University of Genoa, Department of Mechanical Engineering \\ ${ }^{c}$ Johns Hopkins University, Department of Mechanical Engineering \\ ${ }^{d}$ University of Cambridge, Department of Engineering
}

\begin{abstract}
The paper presents a set of one-degree-of-freedom overconstrained linkages, which can be folded into a bundle and deployed into a polygon on a plane. The proposed mechanisms are movable Bricard octahedra of Type III, characterized by the existence of two configurations where all joints are coplanar. The possible geometries of doubly-collapsible Bricard linkages are parameterized and their kinematics is analyzed. A line-intersection method is proposed to construct a bundlefolding mechanism of this type. Necessary and sufficient conditions are derived for the deployedconfiguration polygon to be a square. Simulation and prototype experiment results validate the analysis and design.
\end{abstract}

Keywords: type III Bricard linkage, bundle folding, deployable mechanism, overconstrained linkages.

\section{Introduction}

A deployable mechanism (DM) is capable of configuration change which dramatically alters its shape and size. DMs have many potential applications, including for the rapid construction of structures both in space, e.g., antennas and telescopes $[1,2,3]$, and on earth, in temporary and emergency architecture. A DM which is able to fold into a bundle is of particular interest: minimal size facilitates storage and transport. The most common DMs are composed of scissor-linkage elements, allowing, with good design, the mechanism to be folded into a bundle and deployed into different shapes $[4,5,6]$.

Recently, spatial overconstrained mechanisms have attracted the interest of designers of DMs. Pellegrino et al. studied a bundle-compacting Bennett linkage [7]. Similar research has also been done on the Myard linkage [8], and the Bricard linkages of types I (line-symmetric) and II (planesymmetric) $[7,9,10,11]$. The deployed shapes of the Bennett, and the Type I and II Bricard linkages, are a rhombus, a hexagon, and a rectangle, respectively.

Since it was proposed, the type III Bricard linkage has attracted relatively less attention from researchers $[12,13,14,15,16,17]$. It has two collapsed configurations, i.e., all the six revolute joint axes (and the faces of the octahedron they define) collapse into a plane in two distinct ways. In this study, we discuss its parametrization and propose a line-intersection method for the construction of a bundle-folding Type III Bricard linkage. In its other flat configuration, the mechanism deploys as a quadrangle, a pentagon, or a hexagon. Comparing with the other overconstrained linkages, the

\footnotetext{
${ }^{*}$ Corresponding Author
} 
type III Bricard linkage proposes less fabricating difficulties since the two joint axes on link each are coplanar. Normal machining can guarantee the manufacturing accuracy easily. Feature of bundlefolding encourages the type III Bricard linkage to be used as the basic unit in several potential fields, such as a deployable roof of a transportable canopy, a supporting frame of a deployable antenna, or a large scale deployable grasping manipulator[18]. In addition, by using the kinematic bifurcation of the type III Bricard linkage [17], other potential applications like reconfigurable deployable mechanism can also be achieved [19, 20].

The paper is organized as follows. In the following section, the type III Bricard linkage is reviewed and parameterized. In Sec. 3, a geometric construction is described, demonstrating the existence of bundle-folding linkages of this type. In Sec. 4, for a Bricard bundle with fixed length, the geometric conditions of forming the maximum deployed area are derived. Case studies have been performed on both forward and inverse design, and the obtained mechanisms have been simulated in Sec. 5.

\section{The Type III Bricard Linkage}

Herein we analyze the type III Bricard linkages, which have two collapsible configurations. Due to the special geometric conditions each such mechanism must satisfy, it can be described by only five parameters.

\subsection{Geometric construction}

The Bricard linkage $A B C A^{\prime} B^{\prime} C^{\prime}$ in Fig. 1, is of type III with two collapsed states. The linkage can be constructed as follows: draw two concentric circles of arbitrary radii; choose two arbitrary points $A$ and $A^{\prime}$ outside of the larger circle; construct the tangents from $A$ and $A^{\prime}$ to the circles and determine their intersections $B, B^{\prime}, C$ and $C^{\prime}$. The lines $B C, B^{\prime} C^{\prime}, B^{\prime} C$ and $B C^{\prime}$ will be tangent to a third concentric circle with radius $r_{t}$. Then, the six triangles $A B C^{\prime}, A B C, A B^{\prime} C, A^{\prime} B^{\prime} C, A^{\prime} B^{\prime} C^{\prime}$, $A^{\prime} B C^{\prime}$ taken in this cyclic order and hinged at their common edges, constitute a deformable six-plate linkage with 1 dof [14].

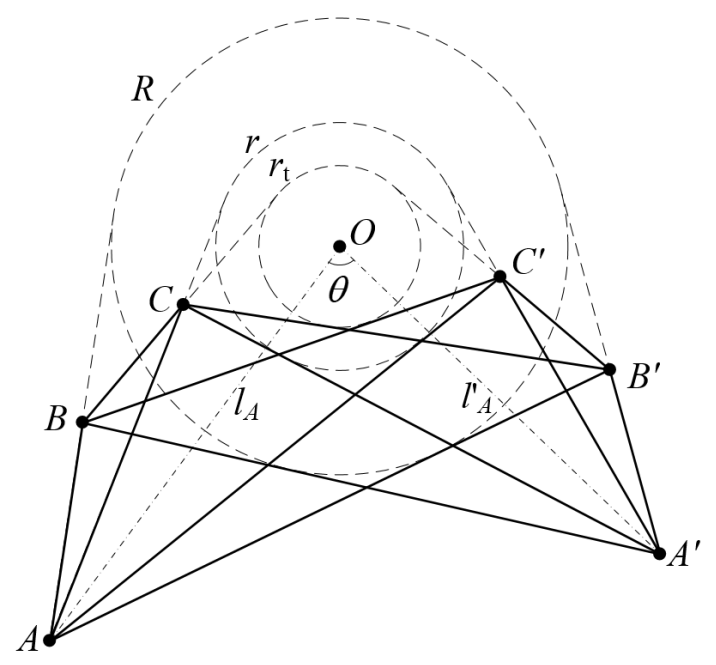

Figure 1: Construction of the type III Bricard linkage

The six triangles in fact define a deformable octahedron: the two remaining (virtual) faces are the triangles $A C B^{\prime}$ and $A^{\prime} C^{\prime} B$, whose shape is constant during the movement. However, if they are physically part of the linkage, link interference is unavoidable. 


\subsection{Parametrization of the Bricard linkage}

The construction in Fig. 1, can be described by the radii of the two circles and the positions of points $A$ and $A^{\prime}$. We denote the radii of the larger and smaller concentric circles by $R$ and $r$, respectively. The lengths of $O A$ and $O A^{\prime}$ are $l_{A}$ and $l_{A}^{\prime}$. The fifth parameter is the angle between $O A$ and $O A^{\prime}$, denoted by $\theta$. A linkage geometry of this type is described completely by the five scalars, $R, r, l_{A}, l_{A}^{\prime}$, and $\theta$. One of the parameters controls the scale of the Bricard linkage; the other four describe the collapsed configuration, which determines the shape and deformation of the octahedron.

\subsection{The two coplanar configurations}

The two collapsible configurations of an example Bricard linkage are shown in Fig. 2. In the figure, the dash-dash-dot lines represent the rotation axes between two triangular panels of the linkage.

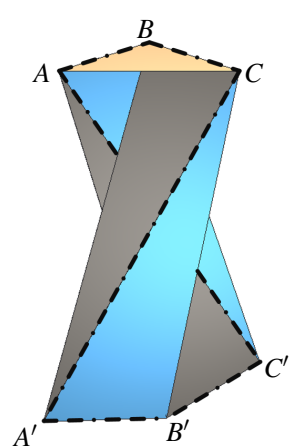

(a)

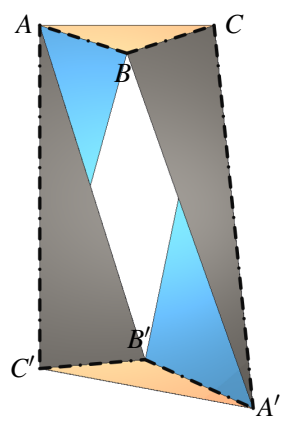

(b)

Figure 2: Two collapsed configurations of a type III Bricard

\section{Bundle-folding Bricard Linkages}

The geometric construction, described above, determines the relationship among the rotating axes which are the most important elements of the mechanism. The six triangles can be seen as connecting bars (physical links) with specific profiles. Kinetics of the linkage will not be affected by altering the geometric outline of the links, but the physical shape of the mechanism will change.

Consider the linkage made with the six triangles mentioned in the first paragraph of Sec. 2. The edges $A B, B C, A C^{\prime}, A^{\prime} B^{\prime}, B^{\prime} C^{\prime}, A^{\prime} C$ are the rotation axes of the mechanism. In Fig. 2(a), draw a line not parallel to any of the six coplanar axes. Then, take the segment of this line connecting the intersection points on any two adjacent R-axes as the physical rigid link. Thus, in this shown configuration, the linkage will be compacted into a line (segment). Once the linkage moves to the other collapsible configuration, as shown in Fig. 2(b), a planar polygon will be formed by those connecting segments. Examples of the segment and the corresponding polygon are illustrated in Fig. 3. Practically, the rigid links have finite thickness and so in the first collapsed configuration, the physical shape of the mechanism will be a bundle; in the second, the top view of the mechanism forms a polygon.

Generally, it is desirable to minimize the distance between the extreme two intersection points on the bundle line, and to maximize the area of the polygon defined by the link segments in the other coplanar configuration. For this reason, one of the two collapsed states is preferred as the bundled configuration, and the other as the deployed one. As can be seen in Fig. 2, the hinge-axis segments (the common triangle edges highlighted with dash-dash-dot lines) are more compactly located in 
Fig. 2(a) than in Fig. 2(b). Therefore, the length of the segment on the bundle line will be shorter in Fig 2(a).

Different choices of the intersecting line result in different deployed shapes and sizes in the second coplanar configuration. Figure 3 gives three examples. The Bricard linkage in Fig. 3 satisfies $A B=B C=A^{\prime} B^{\prime}=B^{\prime} C^{\prime}$.
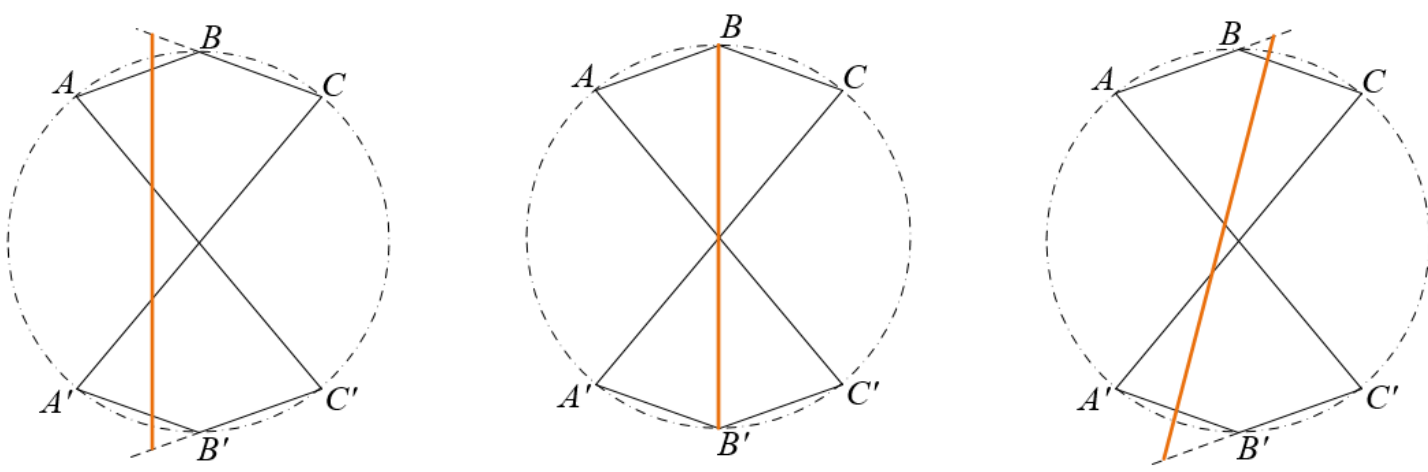

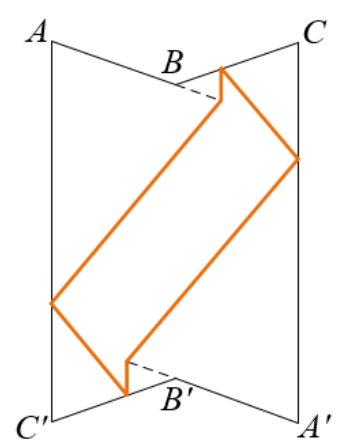

(a)

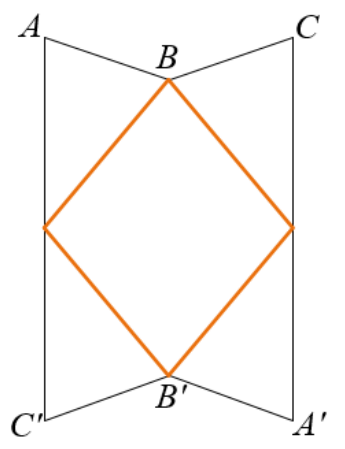

(b)

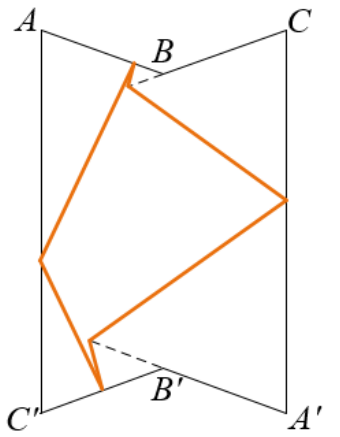

(c)

Figure 3: Bundle-folding linkages form different deployed polygons

The bundle line intersects either a hinge-axis segment or its extension. In Fig. 3(a), the straight line crosses $A B, A C^{\prime}, A^{\prime} B^{\prime}, A^{\prime} C$ and the extensions of $B C$ and $B^{\prime} C^{\prime}$, in addition, it is parallel with $B B^{\prime}$. The formed geometric shape in the other coplanar configuration is a hexagon which has three pairs of parallel sides.

The straight line in Fig. 3(b) is $B B^{\prime}$, intersecting two axes $(A B$ and $B C)$ at $B$, and two others $\left(A^{\prime} B^{\prime}\right.$ and $B^{\prime} C^{\prime}$ ) at $B^{\prime}$. There are only four intersection points of the line with the six axes. Therefore, in the coplanar configuration on the right, the formed shape is a quadrangle.

In Fig. 3(c), the straight line crosses $B C, A C^{\prime}, A^{\prime} C, A^{\prime} B^{\prime}$, and the continuations of $A B$ and $B^{\prime} C^{\prime}$. The formed shape is a hexagon, however, the shape is more general than in Fig. 3(a).

It is obvious that when $B B^{\prime}$ is the segment, which is the case in Fig. 3(b), the formed quadrangle is more regular (a rhombus). Kinematic simulation of this case has been performed, as shown in Fig. 4. A prototype is fabricated to validate the design. In Fig. 3(b), the bundle line forms a quadrangle in the bottom collapsible configuration. In the example in Fig. 4, as the links must have some physical shape and non-zero-area cross-sections, the outline of the mechanism varies a little. In the following analysis, we will only focus on the shape formed by the line, since the link-shape can vary depending on the detailed practical realisation. 

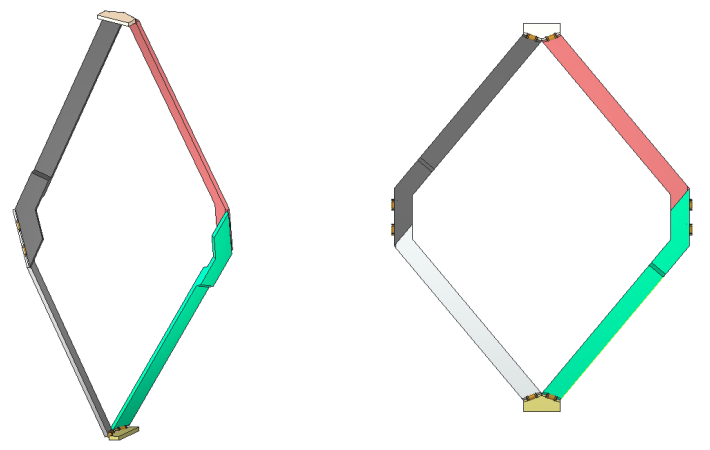

(a) Movement process of the CAD model

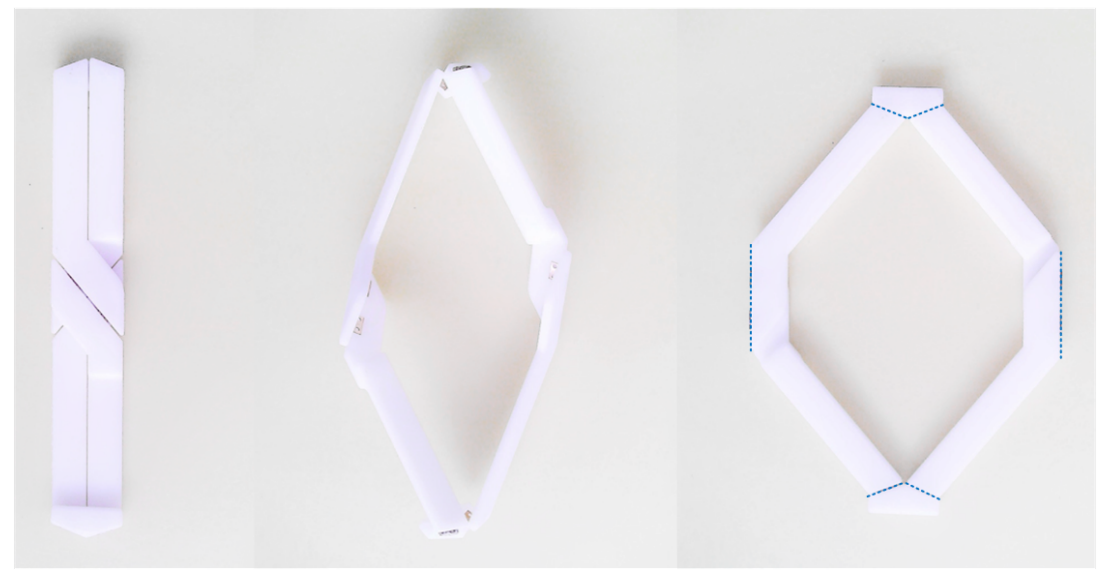

(b) Movement process of the prototype

Figure 4: Kinematic simulation and prototype experiment of a bundle-folding Bricard quadrangle

\section{Deployed Shape Design of the Folded Bundle}

When the intersecting line is along $B B^{\prime}$, the deployed area will be a quadrangle. In this section, the conditions of obtaining a linkage that yields a maximum deployed area are analyzed. Inverse analysis is also performed, obtaining all the parameter sets yielding a bundle-folding Bricard mechanism with a given bundle length. It is well-known that among the quadrangles with the same perimeter, the square has the maximum area. Therefore, we seek the conditions under which the type III Bricard linkage will deploy into a square.

\subsection{Deployable square}

A general type III Bricard linkage is shown in Fig. 5. The bundle line is $B B^{\prime}$. The common point of $B B^{\prime}$ and $A^{\prime} C$ is $D$, while $B B^{\prime}$ and $A C^{\prime}$ intersect at $D^{\prime}$. The six rotation axes are $A B, B C$, $A^{\prime} C, A^{\prime} B^{\prime}, B^{\prime} C^{\prime}$, and $A C^{\prime}$. As the linkage moves, the distance between $B$ and $B^{\prime}$ (which are not on the same face of the octahedron) changes, while points $D$ and $D^{\prime}$ stay on the rigid intervals $A^{\prime} C$ and $A C^{\prime}$, respectively. The deployed quadrangle has its vertices at the positions of $B, D, B^{\prime}$ and $D^{\prime}$ in the alternative collapsed configuration.

Figure 6 illustrates a general bundle-folding Bricard quadrangle together with the construction of the Bricard linkage. The two collapsed configurations are displayed in Fig. 6: $A B C A^{\prime} B^{\prime} C^{\prime}$ and $A B C_{*} A_{*}^{\prime} B_{*}^{\prime} C^{\prime}$, respectively. Triangle $A B C^{\prime}$ is assumed to be the fixed base link. An asterisk denotes the position of a moving point in the second coplanar configuration. The octahedron vertices $B^{\prime}$ and 


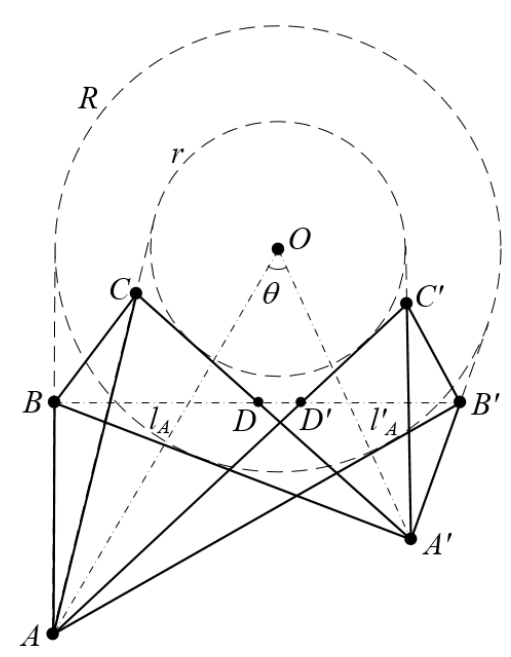

Figure 5: A general Bricard linkage and its $B B^{\prime}$ bundle

$C$ rotate about $A C^{\prime}$ and $A B$, respectively. Therefore, $B^{\prime}$ and $B_{*}^{\prime}$ are symmetric with respect to $A C^{\prime}$, i.e., $\left|B^{\prime} D^{\prime}\right|=\left|B_{*}^{\prime} D^{\prime}\right|$. Similarly, $|B D|=\left|B D_{*}\right|$. The distance between points on the same rigid link does not change. Hence, $\left|B^{\prime} D\right|=\left|B_{*}^{\prime} D_{*}\right|$, as a segment on the rigid panel $A^{\prime} B^{\prime} C$. So, with known length of $B B^{\prime}$ and location of $D$ and $D^{\prime}$, the lengths of the four sides of the quadrangle $B D^{\prime} B_{*}^{\prime} D_{*}$ can be obtained.

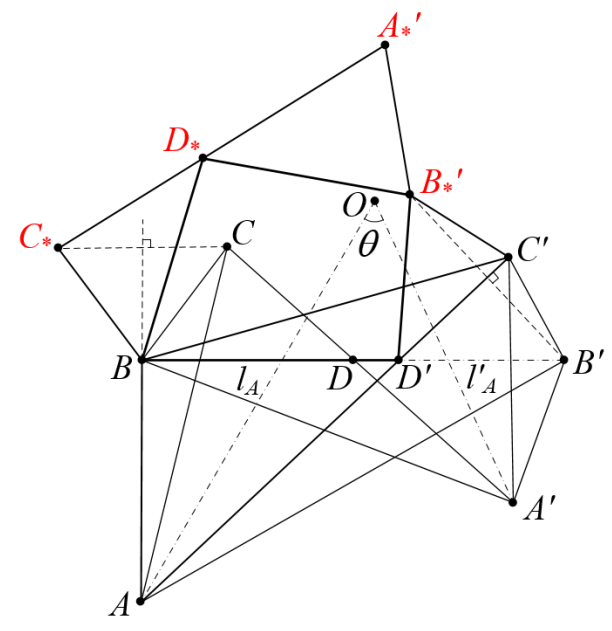

Figure 6: The formed quadrangle and the Bricard linkage

\subsubsection{Geometric conditions}

The Bricard linkage in Fig. 6 must satisfy the following three geometric conditions for the quadrangle $B D^{\prime} B_{*}^{\prime} D_{*}$ to be a square.

(i) $A C^{\prime}, A^{\prime} C$ and $B B^{\prime}$ have a common point, $D=D^{\prime}$

(ii) This intersection, $D$, is at the midpoint of $B B^{\prime}$

(iii) $\angle C^{\prime} D B^{\prime}=45^{\circ}$

(i) $D=D^{\prime} \Leftrightarrow\left|B D^{\prime}\right|=\left|B D_{*}\right| \&\left|B_{*}^{\prime} D^{\prime}\right|=\left|B_{*}^{\prime} D_{*}\right|$ 


\section{Proof}

Sufficiency. Because $B^{\prime} D$ belongs to the rigid body $A^{\prime} B^{\prime} C,\left|B^{\prime} D\right|=\left|B_{*}^{\prime} D_{*}\right|$. Similarly $\left|B^{\prime} D^{\prime}\right|=$ $\left|B_{*}^{\prime} D^{\prime}\right|$, since $B^{\prime} D^{\prime}$ stays on another rigid panel, $A B^{\prime} C^{\prime}$. If $D=D^{\prime}$, then $\left|B^{\prime} D\right|=\left|B^{\prime} D^{\prime}\right|$. Therefore, $\left|B_{*}^{\prime} D_{*}\right|=\left|B_{*}^{\prime} D^{\prime}\right|$, i.e., the two sides of the quadrangle at $B_{*}^{\prime}$ are equal. As $\left|B D_{*}\right|=|B D|$, when $D=D^{\prime}$, $\left|B D_{*}\right|=\left|B D^{\prime}\right|$, i.e., the quadrangle has a kite shape.

Necessity. Obvious: $\left|B D^{\prime}\right|=\left|B D_{*}\right|$ is equivalent to $|B D|=\left|B D^{\prime}\right|$, so $D$ coincidences with $D^{\prime}$.

Once condition (i) is met, there exists one symmetry axis, $B B_{1}^{\prime}$, of the deployed quadrangle. Moreover, when $D=D^{\prime}, O D \perp B B^{\prime}$, as shown in Fig. 7. Proof of the statement is illustrated in Appendix A.

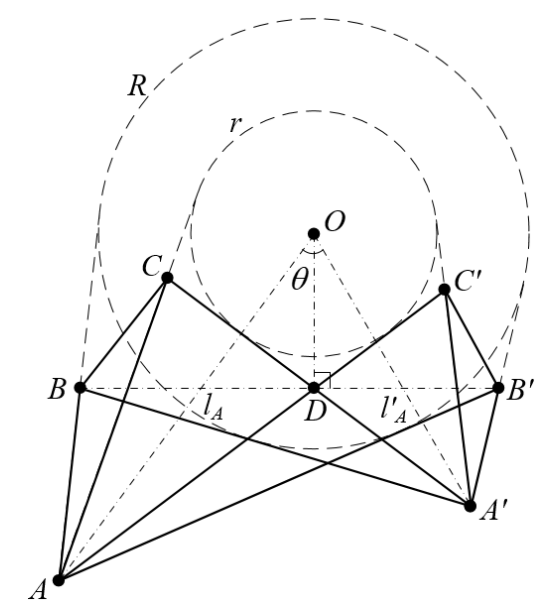

Figure 7: $A C^{\prime}, A^{\prime} C$ and $B B^{\prime}$ intersect at a same point

(ii) $D=D^{\prime}$ is the midpoint of $B B^{\prime} \Leftrightarrow B D^{\prime} B_{*}^{\prime} D_{*}$ is a rhombus

Proof

Sufficiency. When, in addition to Condition (i), $D=D^{\prime}$ is the midpoint of $B B^{\prime},|B D|=\left|B^{\prime} D\right|$, and so $|B D|=\left|B_{*}^{\prime} D\right|$. The four sides of the quadrangle are equal and $B D^{\prime} B_{*}^{\prime} D_{*}$ is a rhombus.

Necessity. If $B D^{\prime} B_{*}^{\prime} D_{*}$ is a rhombus, then its four sides are equal, and so are $|B D|$ and $\left|B^{\prime} D\right|$. Therefore, $D$ and $D^{\prime}$ coincide and divide $B B^{\prime}$ in two equal segments.

By adding constraint (ii), a second mirror-symmetry axis is defined, Fig. 8. So the quadrangle has four equal edge length, in other words, it is a rhombus.

(iii) $\angle C^{\prime} D B^{\prime}=45^{\circ} \Leftrightarrow B D^{\prime} B_{*}^{\prime} D_{*}$ is a square

Proof

Sufficiency. Since the rigid panel $A B^{\prime} C^{\prime}$ rotates around axis $A C^{\prime}$ in Fig. $8, \angle B^{\prime} D B_{*}^{\prime}=2 \angle C^{\prime} D B^{\prime}$ in the other coplanar configuration, and $\angle B D B_{*}^{\prime}=180^{\circ}-\angle B^{\prime} D B_{*}^{\prime}$. When $\angle C^{\prime} D B^{\prime}=45^{\circ}, \angle B^{\prime} D B_{*}^{\prime}$ is a right angle. $B D^{\prime} B_{*}^{\prime} D_{*}$ is a square as it is a rhombus that includes a right angle (see Fig. 9).

Necessity. Obvious.

\subsubsection{Dependence of the geometric parameters}

With the above three geometric conditions satisfied, only two of the five Bricard-geometry parameters are independent. In the following, the relationships among the five geometric parameters of the Bricard linkage are derived. 


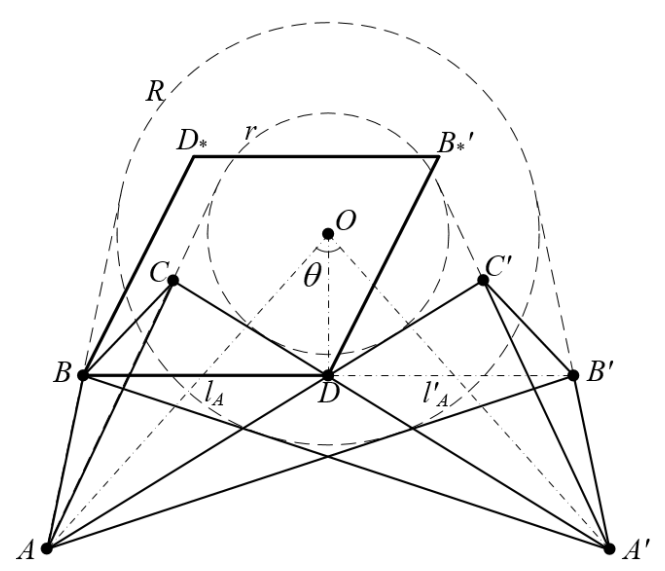

Figure 8: The formed rhombus

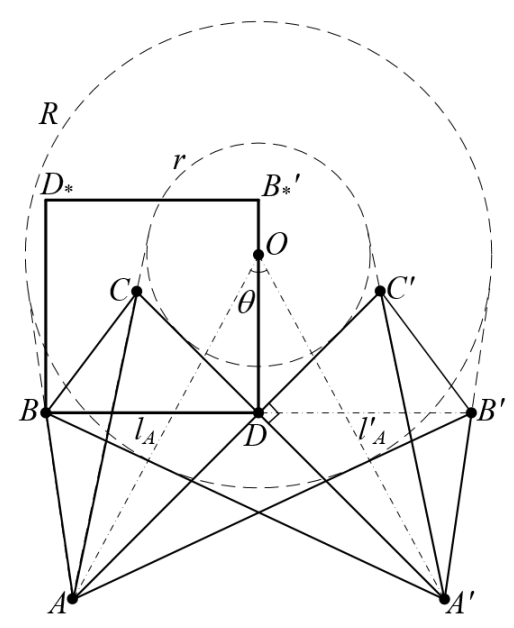

Figure 9: The formed square

First, conditions (i) and (ii) are assumed. We show that then there are three independent parameters determininng the mechanism geometry.

When $D=D^{\prime}$ and $|B D|=\left|D B^{\prime}\right|$, points $B$ and $B^{\prime}$ are symmetric with respect to $O D$, Fig. 8. Due to $O D \perp B B^{\prime}$, we have $|O B|=\sqrt{|O D|^{2}+|B D|^{2}}=\left|O B^{\prime}\right|=\sqrt{|O D|^{2}+\left|B^{\prime} D\right|^{2}}$. It can be concluded, from the construction of the type III Bricard linkage, that $A$ and $A^{\prime}$ are located on the intersection of the tangent lines from $B$ and $B^{\prime}$, which are also symmetric with respect to $O D$. In Fig. 10, there are two pairs of points of tangency: $P_{1}, P_{2}$ and $P_{1}^{\prime}, P_{2}^{\prime}$, on the tangents from $B$ and $B^{\prime}$, respectively.

The angles $\angle P_{2} O B$ and $\angle P_{2}^{\prime} O B^{\prime}$ are, respectively,

$$
\begin{aligned}
& \cos \beta_{1}=\frac{R}{|O B|} \\
& \cos \beta_{2}=\frac{R}{\left|O B^{\prime}\right|}
\end{aligned}
$$

where $\beta_{1}, \beta_{2} \in\left[0,180^{\circ}\right]$. Because $|B D|=\left|B^{\prime} D\right|$, we have $\beta_{1}=\beta_{2}$.

If $\angle P_{1} O P_{1}^{\prime}=\phi$, then $\angle P_{2} O P_{1}^{\prime}=2 \beta_{1}+\phi$ and $\angle P_{2}^{\prime} O P_{1}=2 \beta_{2}+\phi$, which gives

$$
\angle P_{2} O A=\angle P_{2}^{\prime} O A^{\prime}=\frac{2 \beta_{1}+\phi}{2}=\delta
$$




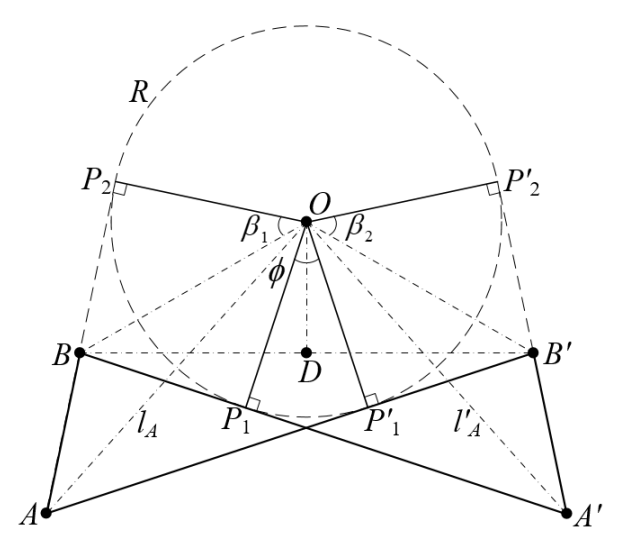

Figure 10: $D$ locates in the middle of $B B^{\prime}$

Since $R=l_{A} \cos \delta=l_{A}^{\prime} \cos \delta$,

$$
l_{A}=l_{A}^{\prime}
$$

In the following, we derive $R$ as a function of $l_{A}, \theta$ and $r$.

From $l_{A}=l_{A}^{\prime}$ we have $\angle O A B^{\prime}=\angle O A^{\prime} B^{\prime}$. So,

$$
\kappa_{1}+\kappa_{2}=\kappa_{1}+\angle O A B^{\prime}+\kappa_{2}-\angle O A^{\prime} B^{\prime}=\angle O A A^{\prime}+\angle O A^{\prime} A .
$$

where $\kappa_{1}=\angle B^{\prime} A A^{\prime}$ and $\kappa_{2}=\angle B^{\prime} A^{\prime} A$. Therefore, $\kappa=\pi-\kappa_{1}-\kappa_{2}=\theta$, which means that points $O$, $A, A^{\prime}, B^{\prime}$ are on the same circle, and so is $B$.

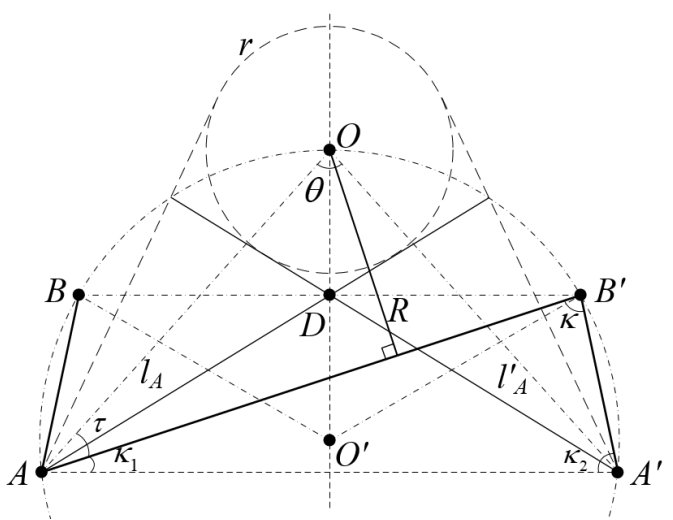

Figure 11: Relationship among $l_{A}, \theta$ and $r$

Because $A$ and $A^{\prime}$ are symmetric with respect to the line $O D$, the latter must pass through the center, $O^{\prime}$ of this circle. For its diameter, we have

$$
d=\frac{l_{A}}{\cos \frac{\theta}{2}}
$$

From the circle with radius $r$,

$$
|O D|=\frac{r}{\sin \left(\frac{\theta}{2}+\arcsin \frac{r}{l_{A}}\right)}
$$


we have

$$
\left|O^{\prime} D\right|=\frac{1}{2} d-|O D|
$$

and

$$
\angle D O^{\prime} B=\arccos \frac{\left|O^{\prime} D\right|}{\frac{1}{2} d}=\arccos \left(1-\frac{2 r \cos \frac{\theta}{2}}{\sin \frac{\theta}{2} \sqrt{l_{A}^{2}-r^{2}}+\cos \frac{\theta}{2} r}\right)
$$

Because all the points are located on a circle, the central angle $\angle B O^{\prime} B^{\prime}$ is 2 times of the angle of circumference $\angle O A B^{\prime}$. So

$$
\tau=\angle O A B^{\prime}=\frac{1}{2} \arccos \left(1-\frac{2 r \cos \frac{\theta}{2}}{\sin \frac{\theta}{2} \sqrt{l_{A}^{2}-r^{2}}+\cos \frac{\theta}{2} r}\right)
$$

Since the circle with radius $R$ is tangent to $A B^{\prime}$,

$$
R=l_{A} \sin \tau
$$

In the second step, all the three conditions from the previous subsection constrain the mechanism. So, $B_{*}^{\prime}$ will locate on $O D$ and $\theta$ becomes a function of $l_{A}$ and $r$,

$$
\theta=90^{\circ}-2 \arcsin \frac{r}{l_{A}}
$$

\subsection{Bricard linkages with the same bundle length}

In the following, we obtain a Bricard linkage that can be deployed into a square for a given length of the bundle.

From the above calculations, the deployed square can be determined by two parameters, e.g., $r$ and $l_{A}$ in (12). There are infinitely many linkages that can be deployed into a square and folded into a bundle with same length. By varying the above equations, the relationship among $r, l_{A}$ and $B B^{\prime}$ is obtained,

$$
\frac{4 \sqrt{2} r l_{A}}{\cos \left(45^{\circ}-\arcsin \frac{r}{l_{A}}\right)}-8 r^{2}=\left|B B^{\prime}\right|^{2}
$$

Therefore, with given $B B^{\prime}$ and $r, l_{A}$ can be calculated with (13). The other parameters can be obtained from (12), (11), and (4).

\section{Case studies}

Two case studies are reported in this section. The first outlines a procedure to obtain a Bricard linkage deployable into a square shape. The second describes several different Bricard linkages with the same bundle length but different geometric parameters. 


\subsection{Case I}

In this case study, we start from a general Bricard linkage, then constrain the deployed shape to a square by adding the constraints step by step.

We take the Bricard linkage in Fig. 5 as the model. The 5 parameters of the initial mechanism are $R=35 \mathrm{~mm}, r=20 \mathrm{~mm}, l_{A}=70 \mathrm{~mm}, l_{A}^{\prime}=50 \mathrm{~mm}, \theta=55^{\circ}$. The bundle line is $B B^{\prime}$ as usual, the deployed shape is in Fig. 6.

From (4) and (11), adding the first two constraints, we have

$$
\begin{array}{r}
l_{A}=l_{A}^{\prime}=50 \mathrm{~mm} \\
R=33.77 \mathrm{~mm}
\end{array}
$$

The bundle and the corresponding deployed rhombus are shown in Fig. 12. When $\theta=90^{\circ}-$ $2 \arctan \frac{r}{l_{A}}=42.84^{\circ}$, the obtained shape is a square as described in Fig. 13.

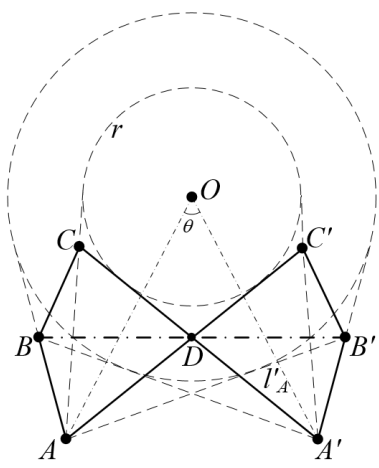

(a)

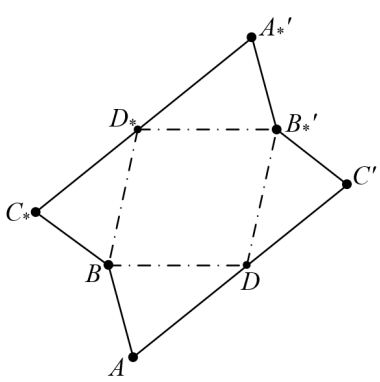

(b)

Figure 12: A Bricard linkage unfolding into a rhombus

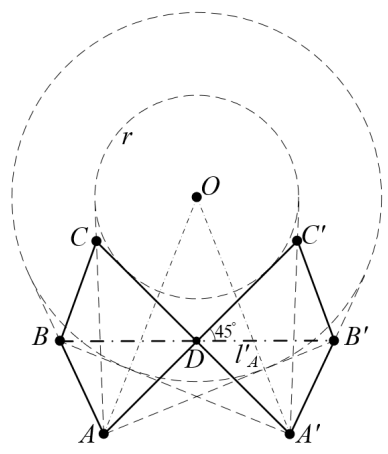

(a)

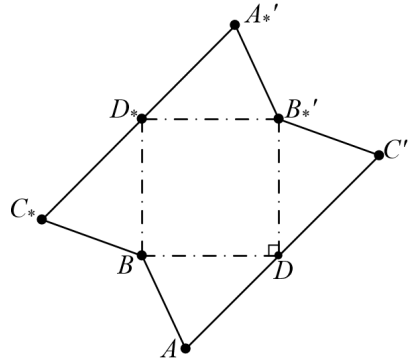

(b)

Figure 13: A Bricard linkage unfolding into a square

\subsection{Case II}

With the same given length $\left|B B^{\prime}\right|=200 \mathrm{~mm}$ and different values $r$, several Bricard linkages are obtained, each of them deployable into a square.

When $r=110 \mathrm{~mm}$, from (13), the other parameters are calculated as $l_{A}=l_{A}^{\prime}=213.35 \mathrm{~mm}$, $\theta=27.93^{\circ}, R=179.47 \mathrm{~mm}$. The construction of the Bricard linkage and the formed square is shown in Fig. 14. 


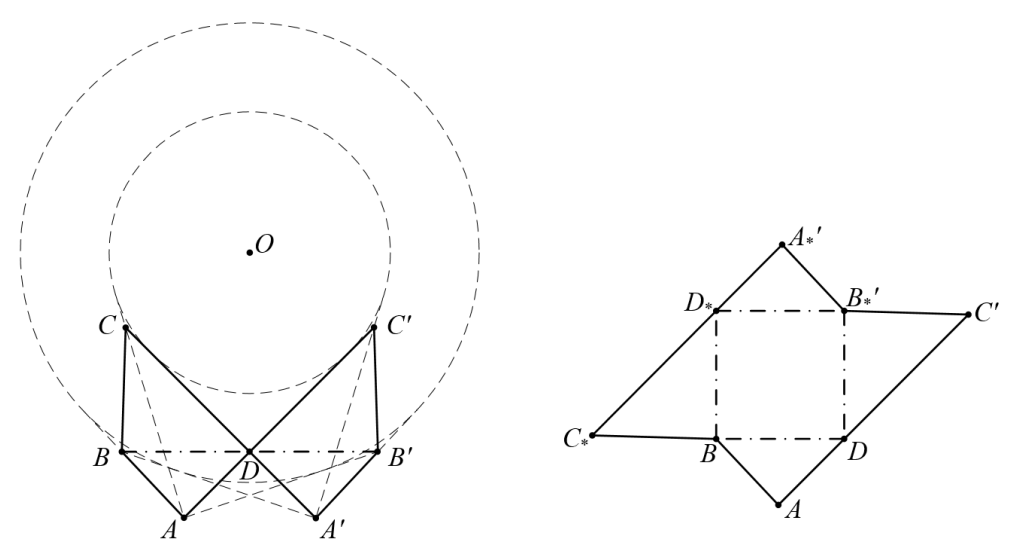

Figure 14: Construction of the Bricard linkage with $B B^{\prime}=200 \mathrm{~mm}$ and $r=110 \mathrm{~mm}$

Next, we use $r=70.71 \mathrm{~mm}, l_{A}=184.78 \mathrm{~mm}, \theta=45^{\circ}$, and $R=130.66 \mathrm{~mm}$. This is a special case, since $D$ and $D_{*}$ are midpoints of $A C^{\prime}$ and $A_{*}^{\prime} C_{*}$ respectively, Fig. 15.
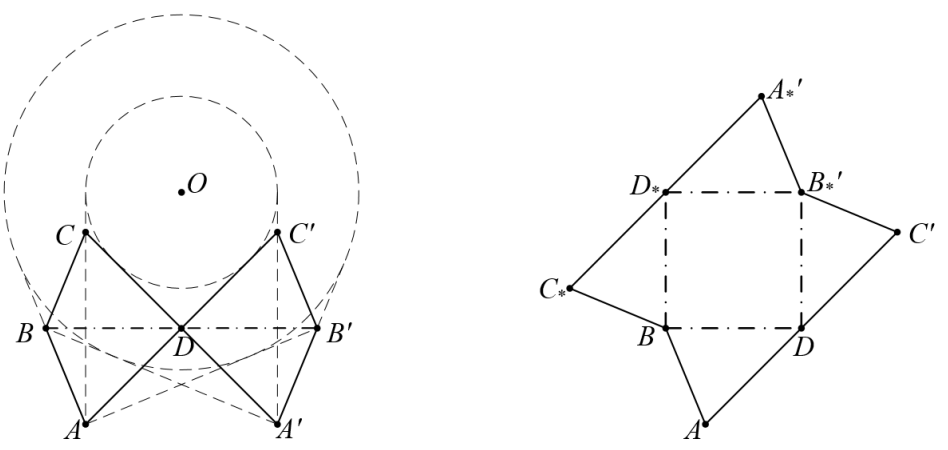

Figure 15: Construction of the Bricard linkage with $B B^{\prime}=200 \mathrm{~mm}$ and $r=70.71 \mathrm{~mm}$

When $r=45 \mathrm{~mm}, l_{A}=188.82 \mathrm{~mm}, \theta=62.42^{\circ}$, and $R=97.87 \mathrm{~mm}$, the two collapsed configurations are shown in Fig. 16.
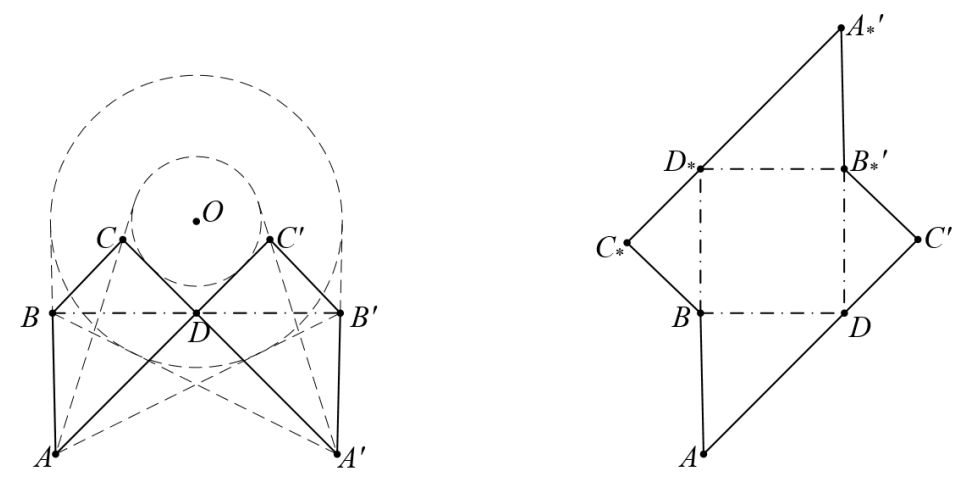

Figure 16: Construction of the Bricard linkage with $B B^{\prime}=200 \mathrm{~mm}$ and $r=45 \mathrm{~mm}$

It can be seen that, under the condition that the bundle length is constant, the smaller the value of $r$, the nearer point $D_{*}$ is to $C_{*}$. When $r=\frac{\sqrt{2}}{4}\left|B B^{\prime}\right|, D$ is in the midpoint of $A^{\prime} C$ and $A C^{\prime}$.

A 3D printed prototype of the Bricard linkage in Fig. 14 has been fabricated where the cross section of each bar is nearly a rectangle, but slightly modified to avoid collisions during the motion. 
Kinematic experiment of the movement of the bundle-folding Bricard linkage is performed as shown in Fig. 17. The formed square is marked with a dotted line in Fig. 17(c).

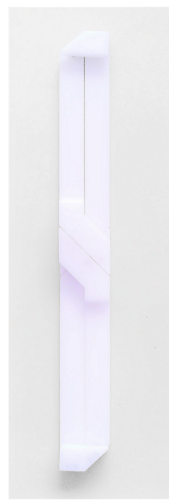

(a)

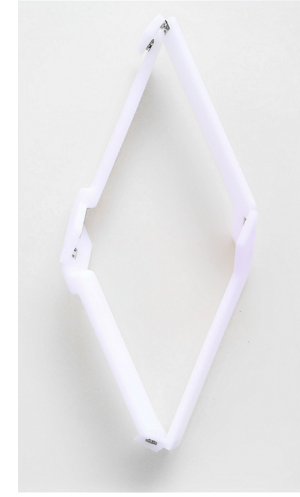

(b)

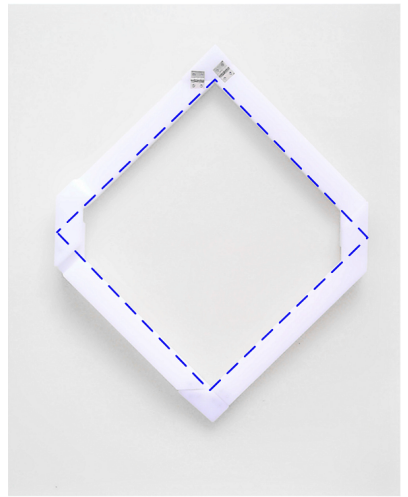

(c)

Figure 17: Prototype experiment of a bundle-folding Bricard square

\section{Conclusion}

Using the capability of the type III Bricard linkage to be coplanar in two configurations, a family of mechanisms has been proposed, each of which can be folded into a bundle and deployed into a planar polygon. The functional relationships among the bundle length, the deployed shape, and the parameters of the Bricard linkage have been analyzed, and the geometric conditions for the construction of a deployable square have been derived. Case studies have been performed, presenting the procedure of obtaining a deployed square, with several choices of the Bricard linkage with the same bundle length and deployed shape.

Although the paper focuses on the construction of deployable quadrangles, with a different choice of the bundle line the mechanism can be also deployed as a hexagon. The proposed lineintersection method can also be applied on other linkages to obtain a bundle folding mechanism.

\section{Acknowledgement}

This research has been supported by the National Natural Science Foundation of China under Grant 51605011, 51635002(Key Program), the Fundamental Research Funds for the Central Universities (YWF-19-BJ-J-336), the State Key Laboratory of Robotics and System (HIT), and the AUTORECON project funded under the Seventh Framework Program of the European Commission (Collaborative Project NMP-FOF-2011-285189). The authors gratefully acknowledge the supporting agencies.

\section{Appendix A}

Consider the construction in Fig. 5, in the following, every line in the figure will be parameterized. The coordinates of their intersections (the points $B, B^{\prime}, D$, and $D^{\prime}$ ) as a function of the coordinates of the points $A$ and $A^{\prime}$ and the radii $r$ and $R$ are computed.

Without loss of generality, take the origin, $O$, of the coordinate system to be at the center of the concentric circles of radius $r$ and $R$ such that $A$ and $A^{\prime}$ respectively lie in the third and fourth quadrants. 
Recall that the line connecting points $P$ and $Q$ can be parameterized as

$$
\mathbf{L}_{P Q}(t)=\mathbf{P}+t(\mathbf{P}-\mathbf{Q})
$$

where $\mathbf{P}$ and $\mathbf{Q}$ are the position vectors of $P$ and $Q$ relative to a coordinate system with origin at $O$. Given two such lines defined by pairs of points $(P, Q)$ and $\left(P^{\prime}, Q^{\prime}\right)$, the coordinates of the intersection of these lines relative to a coordinate frame attached at $O$ can be found by solving the equation

$$
\mathbf{L}_{P Q}(t)=\mathbf{L}_{P^{\prime} Q^{\prime}}\left(t^{\prime}\right)
$$

for the parameters $t$ and $t^{\prime}$.

Explicitly, this gives

$$
\mathbf{P}+t(\mathbf{P}-\mathbf{Q})=\mathbf{P}^{\prime}+t^{\prime}\left(\mathbf{P}^{\prime}-\mathbf{Q}^{\prime}\right)
$$

or

$$
\left(\begin{array}{c}
t \\
t^{\prime}
\end{array}\right)=\left[\mathbf{P}-\mathbf{Q}, \mathbf{Q}^{\prime}-\mathbf{P}^{\prime}\right]^{-1}\left(\mathbf{P}^{\prime}-\mathbf{P}\right) .
$$

The point of intersection is then

$$
\begin{aligned}
\mathbf{X} & =\mathbf{L}_{P Q}\left(\mathbf{e}_{1}^{T}\left[\mathbf{P}-\mathbf{Q}, \mathbf{Q}^{\prime}-\mathbf{P}^{\prime}\right]^{-1}\left(\mathbf{P}^{\prime}-\mathbf{P}\right)\right) \\
& =\mathbf{L}_{P^{\prime} Q^{\prime}}\left(\mathbf{e}_{2}^{T}\left[\mathbf{P}-\mathbf{Q}, \mathbf{Q}^{\prime}-\mathbf{P}^{\prime}\right]^{-1}\left(\mathbf{P}^{\prime}-\mathbf{P}\right)\right) .
\end{aligned}
$$

This equation will be used multiple times.

Let

$$
\mathbf{A}=\|\mathbf{A}\| \mathbf{u}_{A}, \mathbf{r}=\|\mathbf{r}\| \mathbf{u}_{r} \text { and } \mathbf{R}=\|\mathbf{R}\| \mathbf{u}_{R}
$$

respectively denote vectors from the origin to $A$ and to points on the circles of radius $\|\mathbf{r}\|=r$ and $\|\mathbf{R}\|=R$. Without loss of generality, each unit vector in the above expressions can be taken to be of the form

$$
\mathbf{u}(\theta)=\left(\begin{array}{c}
\cos \theta \\
\sin \theta
\end{array}\right)
$$

where $\theta$ is given a subscript that matches that of $\mathbf{u}$.

The relative position vector from a point $\mathbf{R}$ on the circle of radius $R$ to $A$ is

$$
\mathbf{p}=\mathbf{A}-\mathbf{R}
$$

There are only two lines that pass through $A$ and are tangent to the circle of radius $R$. These points of tangency on the circle are defined as $\mathbf{R}_{\text {left }}^{A}$ and $\mathbf{R}_{\text {right }}^{A}$.

The condition that the line between each of these points is tangent to the circle is that

$$
\left(\mathbf{A}-\mathbf{R}^{A}\right) \cdot \mathbf{R}^{A}=0
$$

for both the subscript 'left' and 'right'.

In terms of unit vectors and angles that is

$$
\cos \left(\theta_{A}-\theta_{R}\right)=\mathbf{u}_{A} \cdot \mathbf{u}_{R}=\frac{\|R\|}{\|A\|} .
$$

The angular difference $\left|\theta_{A}-\theta_{R}\right|$ will always be less than $\pi / 2$ because it will be an interior angle in 


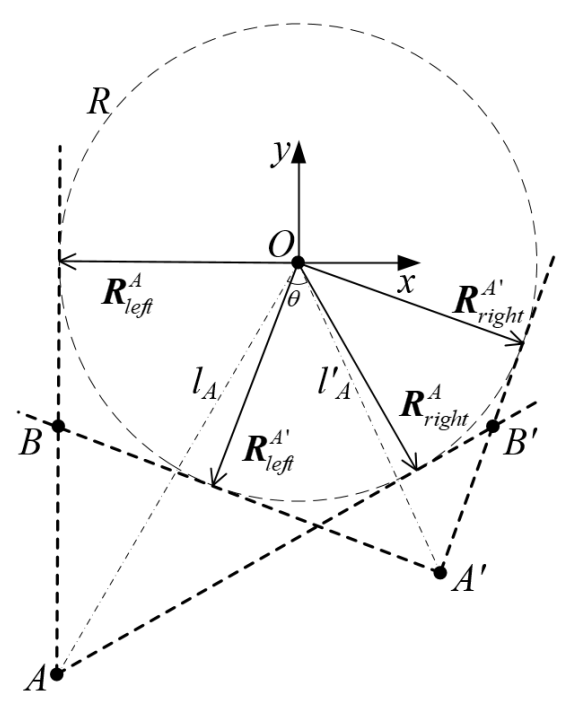

Figure 18: Illustration of when $\theta_{A}-\theta_{R}$ is positive and negative

the triangle formed by $O, A$, and the point of tangency. However, the sign of $\theta_{A}-\theta_{R}$ will depend on which side of the line $O A$ the point $\mathbf{R}$ lies in. If $\mathbf{R}$ is to the left, then this difference will be positive. If it is to the right, it will be negative. Let $\mathbf{R}_{\text {right }}^{A}$ and $\mathbf{R}_{\text {left }}^{A}$ denote these two scenarios, as depicted in Fig. 18.

Consequently, since $\theta_{A},\|R\|=R$ and $\|A\|$ are all known, we can find $\mathbf{R}$ once $\theta_{R}$ is obtained from one of the two formulas

$$
\theta_{R}^{l e f t}=\theta_{A}-\cos ^{-1}\left(\frac{R}{\|\mathbf{A}\|}\right) \text { and } \theta_{R}^{\text {right }}=\theta_{A}+\cos ^{-1}\left(\frac{R}{\|\mathbf{A}\|}\right) .
$$

From here the points $\mathbf{R}_{l e f t}$ and $\mathbf{R}_{\text {right }}$ are obtained from

$$
\mathbf{R}_{\text {left }}^{A}=R \mathbf{u}\left(\theta_{R}^{l e f t}\right) \text { and } \mathbf{R}_{\text {right }}^{A}=R \mathbf{u}\left(\theta_{R}^{\text {right }}\right) .
$$

Everything mentioned above for the large circle of radius $R$ also applies for the small circle of radius $r$, culminating in

$$
\mathbf{r}_{\text {left }}^{A}=r \mathbf{u}\left(\theta_{r}^{l e f t}\right) \text { and } \mathbf{r}_{\text {right }}^{A}=r \mathbf{u}\left(\theta_{r}^{r i g h t}\right) .
$$

In Fig. 5, $A$ is in quadrant 3 and $A^{\prime}$ is in quadrant 4 . The line passing through $A$ and $B$ is the same as the one passing through $\mathbf{A}$ and $\mathbf{R}_{\text {left }}^{A}$. The line passing through $A^{\prime}$ and $B$ is the same as that passing through $\mathbf{A}^{\prime}$ and $\mathbf{R}_{\text {left }}^{A^{\prime}}$. Then using (17), the point of intersection, which is $\mathbf{B}$, is computed as

$$
\mathbf{B}=\mathbf{L}_{\mathbf{A}, \mathbf{R}_{\text {left }}^{A}}\left(\mathbf{e}_{1}^{T}\left[\mathbf{A}-\mathbf{R}_{\text {left }}^{A}, \mathbf{R}_{\text {left }}^{A^{\prime}}-\mathbf{A}^{\prime}\right]^{-1}\left(\mathbf{A}^{\prime}-\mathbf{A}\right)\right) .
$$

Similarly, the position of the point $B^{\prime}$ can be found as

$$
\mathbf{B}^{\prime}=\mathbf{L}_{\mathbf{A}^{\prime}, \mathbf{R}_{\text {right }}^{A^{\prime}}}\left(\mathbf{e}_{1}^{T}\left[\mathbf{A}^{\prime}-\mathbf{R}_{\text {right }}^{A^{\prime}}, \mathbf{R}_{r i g h t}^{A}-\mathbf{A}\right]^{-1}\left(\mathbf{A}-\mathbf{A}^{\prime}\right)\right)
$$

The line between $B$ and $B^{\prime}$ can be parameterized as $\mathbf{L}_{B, B^{\prime}}(t)$. The point where this line intersects the 
line $\mathbf{L}_{\mathbf{A}^{\prime}, \mathbf{r}_{\text {left }}^{\mathbf{A}^{\prime}}}\left(t_{1}\right)$ is $D$. An expression for the coordinates of $D$ can be found using (17). Similarly, the coordinates of $D^{\prime}$ can be found as the intersection between the line $\mathbf{L}_{\mathbf{A}, \mathbf{r}_{r i g h t}^{A}}\left(t_{2}\right)$ and $\mathbf{L}_{B, B^{\prime}}(t)$. This too is calculated using (17).

The expressions for the left side of the statement can be described as

$$
\mathbf{D}=\mathbf{D}^{\prime}
$$

the right side is equivalent to

$$
\mathbf{D} \cdot\left(\mathbf{B}-\mathbf{B}^{\prime}\right)=0 .
$$

Numerical simulations have been performed to prove the equivalence of (28) and (29). In the simulations, values of four parameters $\left(l_{A}, l_{A}^{\prime}, r\right.$, and $\left.\theta\right)$ are set as input. Therefore, the value of $R$ can be solved using (28). It turns out that (29) is always true when substituting the obtained parameters into it. Necessity is proved by value of $R$ solving from (29) can always guarantee (28) is true.

It can also be found from the simulations that there are four independent parameters determining the mechanism geometry when $D=D^{\prime}$ is satisfied.

\section{References}

[1] F. Escrig, J. P. Valcarcel, and J. Sanchez. Deployable cover on a swimming pool in Seville. Bulletin of the International Association for Shell and Spatial Structures, 37(1):39-70, 1996.

[2] J. S. Zhao, J. Y. Wang, F. L. Chu, Z. J. Feng, and J. S. Dai. Mechanism synthesis of a foldable stair. Journal of Mechanisms and Robotics, 4(1):014502, 2012.

[3] G. Durand, M. Sauvage, A. Bonnet, L. Rodriguez, et al. Talc: a new deployable concept for a 20-m far-infrared space telescope. In SPIE Astronomical Telescopes + Instrumentation, pages 91431A-91431A. International Society for Optics and Photonics, 2014.

[4] F. Maden, K. Korkmaz, and Y. Akgün. A review of planar scissor structural mechanisms: geometric principles and design methods. Architectural Science Review, 54(3):246-257, 2011.

[5] J. S. Zhao, F. L. Chu, and Z. J. Feng. The mechanism theory and application of deployable structures based on SLE. Mechanism and Machine Theory, 44(2):324-335, 2009.

[6] Y. Yang, Y. Peng, H. Y. Pu, H. J. Chen, X. L. Ding, G. S. Chirikjian, and S. N. Lyu. Deployable parallel lower-mobility manipulators with scissor-like elements. Mechanism and Machine Theory, 135:226-250, 2019.

[7] S. Pellegrino, C. Green, S. D. Guest, and A. Watt. SAR advanced deployable structure. University of Cambridge, Department of Engineering, 2000.

[8] X. Z. Qi, Z. Q. Deng, B. Li, R. Q. Liu, and H. W. Guo. Design and optimization of large deployable mechanism constructed by Myard linkages. CEAS Space Journal, 5(3-4):147-155, 2013.

[9] Y. Chen, Z. You, and T. Tarnai. Threefold-symmetric Bricard linkages for deployable structures. International journal of solids and structures, 42(8):2287-2301, 2005. 
[10] J. Cui, H. L. Huang, B. Li, and Z. Q. Deng. A novel surface deployable antenna structure based on special form of Bricard linkages. In Advances in Reconfigurable Mechanisms and Robots I, pages 783-792. Springer, 2012.

[11] A. D. Viquerat, T. Hutt, and S. D. Guest. A plane symmetric 6R foldable ring. Mechanism and Machine Theory, 63:73-88, 2013.

[12] R. Bricard. Mémoire sur la théorie de l'octaèdre articulé. Journal de Mathématiques pures et appliquées, pages 113-148, 1897.

[13] A. V. Bushmelev and I. Kh. Sabitov. Configuration spaces of Bricard octahedra. Journal of Mathematical Sciences, 53(5):487-491, 1991.

[14] M. Goldberg. Linkages polyhedral. National Mathematics Magazine, 16(7):323-332, 1942.

[15] J. E. Baker. On the skew network corresponding to Bricard's doubly collapsible octahedron. Proceedings of the Institution of Mechanical Engineers, Part C: Journal of Mechanical Engineering Science, 223(5):1213-1221, 2009.

[16] S. N. Lu, D. Zlatanov, X. L. Ding, M. Zoppi, and S. D. Guest. A network of type III Bricard linkages. Journal of Mechanisms and Robotics, 11(1):011013, 2019.

[17] S. N. Lu, D. Zlatanov, X. L. Ding, M. Zoppi, and S. D. Guest. Reconfigurable chains of bifurcating type III Bricard linkages. In Advances in Reconfigurable Mechanisms and Robots II, pages 3-14. Springer, 2016.

[18] G. L. Jia, H. L. Huang, B. Li, Y. L. Wu, Q. D. Cao, and H. W. Guo. Synthesis of a novel type of metamorphic mechanism module for large scale deployable grasping manipulators. Mechanism and Machine Theory, 128:544-559, 2018.

[19] R. M. Li, Y. A. Yao, and X. W. Kong. A class of reconfigurable deployable platonic mechanisms. Mechanism and Machine Theory, 105:409-427, 2016.

[20] J. Y. Wang and X. W. Kong. Deployable mechanisms constructed by connecting orthogonal Bricard linkages, 8r or 10r single-loop linkages using s joints. Mechanism and Machine Theory, 120:178-191, 2018. 